\title{
RETOS Y PERSPECTIVAS DE LA UNIVERSIDAD TÉCNICA DE MACHALA ANTE LA TRANSFORMACIÓN CURRICULAR DE LA EDUCACIÓN SUPERIOR ECUATORIANA
}

\author{
Elida Rivero Rodríguez ${ }^{1}$, Amarilis Borja Herrera \\ Oscar Riofrío Orozco \\ Universidad Técnica de Machala. (Ecuador) ${ }^{1}$ \\ erivero@utmachala.edu.ec
}

Cómo citar este artículo: Rivero, E., Borja, A. y Riofrío, O. (2015) Retos y perspectivas de la Universidad Técnica de Machala ante la transformación curricular de la Educación Superior Ecuatoriana. CUMBRES, Revista Científica. 1 (1) 24 - 29

\section{RESUMEN}

La tendencia actual de la Educación Superior ecuatoriana es el perfeccionamiento continuo, conducente a la preparación del hombre para la vida. En tal sentido, los modelos académicos deben considerar las tendencias epistemológicas del conocimiento a nivel latinoamericano y mundial, para hacer de las universidades instituciones pertinentes. Para ello, los cambios que deben operarse han de integrar la transición del conocimiento disciplinar, al inter y transdisciplinar; de la homogeneidad de actores y espacios de aplicación del conocimiento, a la heterogeneidad; de los circuitos de producción del conocimiento cerrados y descontextualizados, a circuitos abiertos y en contextos de aplicación. Frente a este desafío la dirección académica de la UTMach diseño un proyecto de mejoramiento académico, los resultados de su avance se presentan a partir del análisis de los retos y perspectivas que se afrontan para responder al imperativo de la Educación Superior ecuatoriana ante la transformación curricular de sus programas académicos.

Palabras clave: Transformación curricular, conocimiento disciplinar y transdisciplinar

\section{ABSTRACT}

The current trend of the Ecuadorian higher education is continuous improvement, leading to the preparation of men for life. In this regard, the academic models should consider the epistemological understanding trends in Latin America and globally, to make relevant institutions universities. To do this, the changes to be operated have to integrate the transition from disciplinary knowledge, inter and trans-disciplinary; uniformity of actors and areas of application of knowledge, heterogeneity; circuit production closed and decontextualized knowledge, open circuits and application contexts. Faced with this challenge the academic direction of the design UTMach Improvement project, the results of their progress are presented from the analysis of the challenges and perspectives front to respond to the imperative of Ecuadorian higher education to the curriculum transformation their academic programs.

Keywords: Curriculum Transformation, disciplinary and trans-disciplinary knowledge 


\section{INTRODUCCIÓN}

Hasta la primera mitad del pasado siglo XX, era posible afirmar con bastante certeza que cuando una persona culminaba sus estudios universitarios estaba preparada para ejercer profesionalmente durante toda su vida. Hoy no ocurre de ese modo. Ni los conocimientos se atesoran privilegiadamente en la sociedad, ni es posible pensar en tener desempeños profesionales exitosos sin una constante actualización.

Hoy en día la Educación Superior, en todo el mundo, enfrenta retos particularmente difíciles: pues debe formar profesionales capaces no simplemente de adaptarse a los imprevisibles cambios de la sociedad y de las actividades técnicas, científicas y sociales, sino de generar y conducir dichos cambios; encontrando las formas de incidir de manera cada vez más decidida, permanente y eficaz en la sociedad (en todos sus ámbitos); también, y cada vez de manera más amplia, debe superar el economicismo educativo (que reduce la educación a un mero insumo de la producción, y al ser humano a "recursos humanos") y rescatar el valor cultural de la educación.

Al respecto, la universidad ecuatoriana no puede incurrir en evadir responsabilidades, ni restar importancia a los desafíos que la desbordan, puesto que se han levantado justificadas críticas a su accionar, al asumir de manera convincente la superación de los vacíos y paradojas epistemológicas y sobre el rol desempeñado por ellas en el marco general de las tensiones y la crisis sistémica del capitalismo, que como manifiesta (Herrera et al., 2006) ha incidido "en la formación de personas que poseen perfiles multidisciplinarios, y que generan conocimientos en el contexto de su aplicación y se vinculan orgánicamente al entorno, a lo que la sociedad demanda."

Las respuestas son innumerables, a ello han contribuido mucho quienes se han atrevido a formular cambios, sustentando tesis mediante el debate democrático, defendiendo con firmeza las actuales tendencias, en unos casos, con discusiones en cada uno de los claustros universitarios, en otros, acogiendo las líneas y directrices de las Conferencias Latinoamericanas del Instituto de Educación Superior para América Latina y el Caribe, IESALC-UNESCO y la Conferencia Mundial de Educación Superior de la Organización de las Naciones Unidas para la Educación, la Ciencia y la Cultura (UNESCO, 2010), a fin de identificar el diseño y gestión de alternativas a seguir ante las debilidades detectadas en las Instituciones de Educación Superior (IES), las mismas que poseen un valor heurístico en cuanto a la autocrítica e interpretación de sus reales motivos y razones históricas que las producen.

Es decir, sin desestimar la trayectoria recorrida por la universidad ecuatoriana, corresponde ahora profundizar en su capacidad de búsqueda continua e incesante de una nueva filosofía coherente con su vida institucional, empoderando una adecuada comprensión del papel activo que les corresponde asumir a las IES en los procesos de desarrollo social, como instancias innovadoras capaces de inspirar y provocar cambios sociales, y de promover espíritus emprendedores que los lideren. (Larrea, 2014)

En tal sentido, al amparo de las nuevas concepciones el principal reto de las IES, es no permanecer ajenas e inmutables a su realidad, sino efectuar los cambios pertinentes, dentro de la coyuntura emergente y vibrante, sustentados en el diagnóstico exploratorio realizado a nivel de país el cual evidencia los principales problemas que afectan a la organización académica de la Educación Superior ecuatoriana, los cuales han sido referenciados como nodos críticos que se convierten en oportunidades para el cambio necesario.

Entre estos nodos críticos delimitados en el diagnóstico en relación a la organización académica y la propuesta curricular se encuentran: la integralidad del sistema y las trayectorias e itinerarios educativos, la diversificación y organización de los modelos curriculares y de los aprendizajes, la pertinencia del modelo educativo; la validación de habilidades y desempeños de titulación de estudiantes e inserción laboral, la investigación y producción académica y la formación e integración del personal académico; considerándolos nodos críticos a partir de los cuales pueden generarse múltiples oportunidades de intervención para el mejoramiento de la calidad de la Educación Superior.

Lo anterior justifican que los cambios que deben operarse han de integrar la transición del conocimiento disciplinar, al inter y transdisciplinar; de la homogeneidad de actores y espacios de aplicación del conocimiento, a la heterogeneidad; de los circuitos de producción del conocimiento cerrados y descontextualizados, a circuitos abiertos y en contextos de aplicación; y de la aplicación técnica y comercial de los saberes a la "aplicación socialmente edificante y solidaria" (De Souza, 2008).

Bajo estos preceptos, la Educación Superior ecuatoriana se encuentra en una etapa de perfeccionamiento del proceso docente educativo, conducente a la preparación del hombre para la vida.

En tal sentido, los modelos académicos deben considerar: 
los cambios que se operan en los horizontes epistemológicos del conocimiento, las nuevas tendencias de la Educación Superior a nivel latinoamericano y mundial, las reformas académicas, los planes de desarrollo y las visiones y necesidades de los actores y sectores; para hacer de las universidades instituciones pertinentes y de calidad. Proporcionando además ambientes de aprendizaje con claras dinámicas de organización del conocimiento y los saberes, que produzcan espacios sociales y epistemológicos para interpretar los problemas de la ciencia y la realidad, orientados a la implicación con su transformación, en el marco de una formación ciudadana e intercultural.

Lo anterior constituye el problema a resolver en la presente investigación, enunciado en: la necesidad de rediseñar el currículo de la nueva oferta académica de las carreras de la UTMach, en correspondencia con los elementos anteriormente argumentados, delimitando en primer lugar al currículo como:

.....una construcción social y colectiva, fundamentada en un proceso continuo de investigación y evaluación de las tendencias de la ciencia, la sociedad, la profesión $y$ del tejido de interacciones de los actores educativos. Expresa y define los fines de la educación, y promueve un plan de acción que se concreta en un proyecto pedagógico y de formación, critico, dinámico, participativo y creativo, orientado a generar experiencias de aprendizaje que produzcan una aproximación entre el conocimiento, la realidad y la producción de significados del sujeto educativo, desarrollando una serie de saberes y competencias que van incidiendo sobre su identidad personal, profesional y ciudadana, en el marco de un contexto productivo, político, social, ambiental y cultural determinado, propiciando su transformación. (Larrea, 2014).

Por tanto, tendrán como centro de atención al profesional que se quiere formar, en función de las necesidades sociales del entorno y debe ser colegiado con los actores (profesores, estudiantes, empleadores, administrativos, etc.) que se implicaran en su ejecución y evaluación.

Bajo estos preceptos en la Dirección Académica de la Universidad Técnica de Machala (UTMach) se diseñó un proyecto de mejoramiento académico para el componente rediseño de la nueva oferta académica, los resultados de su avance representan el objetivo del presente artículo, el cual se constituye en aportar un análisis pormenorizado de los retos y perspectivas que afronta la UTMach para responder al imperativo de la Educación Superior ecuatoriana ante la transformación curricular de sus programas académicos.

El principal objetivo de la agenda ecuatoriana es edificar un Sistema de Educación Superior cuyo carácter público, esté asegurado para el bien común de las y los ecuatorianos. No obstante, este objetivo primario debe inscribirse dentro de un propósito más profundo, que es coadyuvar a las transformaciones por las cuales la sociedad debe transitar a fin de hacer efectivo y concretar la sociedad del Buen Vivir o Sumak Kawsay (Ramírez, 2010).

En este marco, hay que replantear el sistema cognitivo que se necesita para esa nueva sociedad ecuatoriana que se desea construir y en efecto, si bien la construcción de un sistema de innovación y las transformaciones en el campo de la Educación Superior pueden ser objetivos loables en sí mismos, no son el fin último. Es urgente pensar que las transformaciones en el sistema cognitivo se vuelven necesarias para construir el tipo de sociedad que deseamos. Esto implica construir un sistema de Educación Superior que constitucionalice el proyecto de sociedad firmado por los ecuatorianos y ecuatorianas.

En tal sentido se presentan los resultados del proyecto de mejoramiento académico para el componente diseño de la nueva oferta académica, el cual partió de un análisis pormenorizado de las carreras que actualmente se ofertan en la UTMach y su relación con lo que establece el (RPCSO_27_No. 289-2014), para delimitar su estatus en relación a los campos del conocimiento y la titulación que otorga.

Para ello se utilizaron diferentes técnicas asociadas al método de investigación empírico, las cuales se relacionan a continuación:

- Estudio de los documentos normativos de la Educación Superior ecuatoriana Reglamento de Régimen Académico, Ley Orgánica de Educación Superior (LOES, 2010) y los estatutos de la UTMach.

- Análisis de informes y estudios anteriores desarrollados en la UTMach

- Entrevistas a directivos y docentes de la UTMach para conocer las fortalezas y debilidades en relación al proceso de rediseño curricular.

- Utilización de la técnica de grupos de discusión, para listar de manera colegiada los aspectos que requieren ser mejorados en el proceso docente educativo.

- Capacitación a las comisiones de trabajo nombradas para el rediseño de la nueva oferta académica.

Además el proyecto asumió como población a las 33 carreras en vigencia en las diferentes unidades académicas de la 
UTMach, una vez establecido el Reglamento de armonización de la nomenclatura de títulos profesionales y grados académicos que confieren las Instituciones de Educación Superior del Ecuador (RPC-SO_27_No. 289-2014) se continuó el proceso de rediseño de la nueva oferta académica con 32 a tenor de que la Licenciatura en Economía Agropecuaria no se encuentra listada en el mencionado reglamento.

Como resultado de ese análisis se pudo constatar que de las 33 carreras vigentes:

A 17 carreras le cambia la titulación en el diseño de la nueva oferta académica. De ellas 6 requirieron de un análisis entre dos o más opciones de titulación

2 carreras cambiaron de campos del conocimiento

1 carreras no se encuentra listadas en la (RPC-SO_27. No. 289-2014).

Por tanto, el diseño de la nueva oferta académica actualmente se desarrolla en 32 carreras de las 33 vigentes, iniciándose el trabajo a partir del estudio de pertinencia de las mismas.

\section{ANÁLISIS - DISERTACIÓN}

Para enfrentar la transformación académica de la nueva universidad ecuatoriana se requiere en estos supremos momentos, saber conjugar la inteligencia con la voluntad, para tener la capacidad de comprometernos con una reforma educativa que abarque e incorpore cambios trascendentales en la formación, la investigación y la vinculación universitaria, capaz de provocar revoluciones académicas y sociales en el conocimiento y la información.

Por tanto, es necesario resolver y tomar conciencia de que estamos frente a grandes desafíos de transformación de la organización académica los cuales requieren del debate y accionar de todos los actores del proceso universitario, imprescindible para entender que la Educación Superior, como bien público, constituye un espacio colectivo de realización de los derechos ciudadanos, desde su aporte en la construcción de proyectos territoriales de innovación social, que contribuyen al mantenimiento de un Estado constitucional de derechos, justicia y de bienestar para todos.

Estos desafíos de transformación de la organización académica que a continuación se explicitan, la dirección académica de la UTMach los considera retos a enfrentar:

La ruptura epistemológica: Promueve una nueva forma de organización del conocimiento y los apren- dizajes exigiendo nuevas formas de integración de la ciencia y los saberes, con modelos inter y transdisciplinarios que disuelven fronteras y barreras disciplinares para construir nuevas tecno-ciencias cuyas olas de transformación son estructurales y de largo alcance. La ruptura epistemológica en la UTMach infiere el rediseño de sus carreras a partir del cambio de una formación exclusivamente disciplinar a una forma de organización del conocimiento multi, inter y transdisciplinarios. Justificado en que los modelos disciplinares conciben el estudio de la realidad desde una serie de fragmentaciones, lo que impide integrar conocimientos, componentes, dimensiones y enfoques del conocimiento científico. En tanto los nuevos abordajes del conocimiento de carácter multi, inter y transdisciplinarios rompen con estas formas de comprensión de la ciencia y la realidad, planteando la complejidad en los estilos de pensamiento, las integraciones del conocimiento y los saberes, la multi dimensionalidad en los abordajes interpretativos, la poli causalidad y los efectos recursivos de las tensiones, problemas y oportunidades de las realidades sociales y naturales. Por tanto, está ruptura epistemológica representa un doble reto para los académicos de la UTMach, a partir de que se introduce una nuevo forma de organizar el conocimiento y por otra parte es importante considerar que la inmensa mayoría de los profesores universitarios, no se han formado en instituciones pedagógicas, sino que como producto de su alta calificación profesional, han asumido la tarea de enseñar. Lo cual representa un imperativo de capacitación para asumir esté desafío.

El desarrollo de currículos que posibiliten la integración de las funciones sustantivas universitarias (la formación, la investigación y la gestión social del conocimiento): Para dar respuestas a las demandas de una sociedad cambiante y dinámica, articulación que se sustenta en el desarrollo de capacidades y condiciones para la construcción de dominios tecno-científicos y humanistas, cuya estructura se centra en competencias diferenciadas de saberes, áreas estratégicas de desarrollo y en redes de construcción plural de lo público cuyos significados son producidos desde, por y para los sujetos sociales.

Modelos educativos centrados en aprendizajes conectados, interactivos, en red y en convergencia de medios: En los que cuales, los procesos de aprendizaje y generación de conocimiento se practique la movilidad estudiantil, de profesores e investigadores, tanto al interior del sistema de Educación Superior como del de innovación, pero siempre y cuando se 
articulen con el resto de actores de la sociedad. Esto implica tener una pedagogía de ojos abiertos (sentidos), en donde la sociedad y el medio ambiente sean vistos como aula; la ciudadanía como compañera de clase, y los derechos, necesidades y potencialidades de la humanidad y de la naturaleza como objeto de investigación.

\section{La contextualización y pertinencia de la produc- ción del conocimiento y sus aprendizajes: No se} puede seguir pensando en procesos de formación profesional cuyos saberes están exclusivamente en las mentes de académicos y condicionados por las agendas del mercado, porque estos producen intervenciones que aunque son válidas, no son únicas y tienden a estar centrados en el oficio o descontextualizados. De manera que está nueva episteme, debe propiciar un salto cualitativo hacia la pluriversidad, o a lo que (Morín, 2001) denomina multiversidad. En todo caso es buscar construir una ciencia, que permita a los diferentes actores de la sociedad interactuar y participar con los actores universitarios en la solución de problemas que los afecta directa o indirectamente y como parte del propio proceso de aprendizaje y de construcción de una democracia (plurinacional e intercultural) radicalmente humana y sostenible

\section{La configuración de ambientes de aprendizaje} centrados en la comunicación y la interacción: Significa que los medios, las TICs y las redes sociales deben ser abordados de manera crítica y creativa tomando en cuenta que estamos en un tiempo en que la información es distribuida de forma instantánea, incontenible y sin ningún tipo de control. A tal efecto la formación profesional debe educar en y para el ejercicio de la participación en las redes, proporcionando la inserción en estructuras reticulares y multi nodales (conectadas y con muchos ejes de intersección), lo que permitirá la conexión de experiencias, información, proyectos, ideas y cosmovisiones.

El diálogo intercultural: El conocimiento científico y sus aprendizajes no pueden generarse independientemente de los escenarios en donde se aborda y se construyen interpretaciones de la realidad como los saberes culturales, por lo que desde este postulado el currículo debe convertirse en un escenario educativo para la descolonización del aprendizaje, la validación de la diversidad de pensamientos, enfoques y cosmovisiones y el reconocimiento de las diferentes identidades e itinerarios culturales de los sujetos que aprenden. De manera que al considerar en el proyecto curricular de las carreras el enfoque de interculturalidad, se favorece la formación de una ciudadanía más amistosa con la diversidad y la diferencia, etnias, género, cultura, religión, edad, pensamiento, pues tendrán en los ambientes de aprendizajes un lugar para ser visibilizado, un espacio para participar, crear e innovar sus formas de vida a partir del conocimiento. Por tanto en el diseño de la nueva oferta académica se hace necesario la delimitación de escenarios de aprendizaje que posibiliten el desarrollo de enfoques de género y de interculturalidad que permita el ejercicio de una educación democrática, incluyente y diversa, basada en el diálogo de saberes como sostiene la Constitución de la Republica de Ecuador.

La visualización del proyecto de vida de los estudiantes y docentes: El indispensable que en el rediseño de la nueva oferta académica de las carreras de la UTMach se visualizase el proyecto de vida de los estudiantesy profesores, destacándose los saberes que surgen de sus experiencias y rescatando sus trayectorias, visiones, filiaciones y cultura en los procesos de aprendizaje, en estrecha relación con los objetivos y líneas de desarrollo del Plan Nacional del Buen Vivir (PNBV). Lo anterior revela que las transformaciones en el sistema cognitivo que hoy se vislumbran en la nueva oferta académica, se vuelven necesarias e implican construir un sistema de Educación Superior que constitucionalice el proyecto de sociedad firmado por los ecuatorianos y ecuatorianas. Por tanto, estos retos propician importantes cambios en el diseño de la nueva oferta académica, los cuales se constituyen en perspectivas de desarrollo académico para la UTMach:

El desarrollo de un currículo con procesos de calidad, pertinencia, flexibilidad, transversalidad e integración de saberes, centrado en los sujetos de aprendizaje, y en la generación, difusión y preservación de saberes profesionales y conocimientos científicos tecnológicos y humanistas. Procesos de generación y transferencia de saberes profesionales desarrollados en la investigación, con la incorporación de la innovación tecnológica y el valor productivo, social y cultural del conocimiento.

La promoción de redes de cooperación académica entre instituciones, capaz de integrar las nuevas áreas del conocimiento a modelos de intervención y desarrollo.

La actualización y profundización permanente y sistemática de los campos de estudio y de actuación de la formación profesional de las carreras, a través de la investigación y vinculación con el entorno. La promoción de procesos curriculares transversales que promuevan la interdisciplinariedad, así como principios y valores que contri- 
buyan a la formación de la educación ciudadana.

- La producción de espacios curriculares de innovación productiva y social, así como la vinculación con los organismos de desarrollo de la profesión, para la generación de modelos y metodologías de intervención que impacten en las relaciones políticas, económicas y socio-institucionales de la sociedad del buen vivir.

- Bajo estas perspectivas queda clarificada la finalidad de las transformaciones que en los momentos actuales plantea la Educación Superior ecuatoriana y hacia ellas se direcciona el diseño de la nueva oferta académica de la UTMach.

\section{CONCLUSIONES}

1. El proceso de transformación curricular en el que se encuentra inmerso la Educación Superior ecuatoriana constituye un reto para los académicos de la UTMach, hacia el cual se trabaja visualizando sus perspectivas de mejora en la formación de un profesional con calidad, calidez y pertinencia.

2. El análisis y contextualización de los principales aspectos que incluye el rediseño de la nueva oferta académica, ha favorecido el avance del trabajo de las 32 carreras que actualmente se encuentran insertadas en dicho proceso.

3. La transformación curricular en la que está inmersa la UTMach es una tarea de gran alcance, que demanda tiempo, dedicación y empoderamiento por parte de los profesores, de los aspectos esenciales del nuevo modelo que se pretende implantar.

\section{REFERENCIAS BIBLIOGRÁFICAS}

- De Souza, B. (2008). El Rol de la Universidad en la Construcción de una Globalización Alternativa. En Taylor Peter (Ed), La Educación Superior en el Mundo, Global University Network for Innovation, Madrid: Mundi-Prensa.

- Herrera, A. y Didriksson, A. (2006). Manual de planeación prospectiva estratégica. Su aplicación a Instituciones de Educación Superior. México: CESU-UNAM.

- Larrea, E. (2014). El currículo de la Educación Superior desde la complejidad sistémica. Algunas consideraciones para orientar el proceso de construcción del nuevo modelo de formación universitaria. Ecuador: Consejo de Educación Superior.

- Ley Orgánica de Educación Superior. (2010). Quito: Presidencia de la República. Oficio No. f. 4454-SNJ-10-1512.
LOES.

- Morín, E. (2001). Introducción al pensamiento complejo. Barcelona: Editorial Gedisa.

- Ramírez, R. (2010). "Introducción"; en René Ramírez (coord.): Transformar la Universidad para Transformar la Sociedad. Quito: SENPLADES.

- Ramírez, R. y Minteguiaga, A. (2010). "Transformaciones en la Educación Superior Ecuatoriana: Antecedentes y perspectivas futuras como consecuencias de la nueva constitución política". Revista Educación Superior y Sociedad, No 1, UNESCO.

- Reglamento de armonización de la nomenclatura de títulos profesionales y de grados académicos que confieren las Instituciones de Educación Superior del Ecuador. RPC-SO_27_No. 289-2014. Ecuador: CES. 